\title{
Laparoscopic Ventral Hernia Repair
}

\author{
Youngeun Park, M.D., Min Chung, M.D., Ph.D. \\ Department of Trauma Surgery, Gacheon University Gil Medical Center, Incheon, Korea
}

\begin{abstract}
Laparoscopic ventral hernia repair is performed less frequently than open repair because some ventral hernias are unsuitable for laparoscopic repair and the complications are more severe than those of open repair. However, currently, the incidence of laparoscopic hernia surgery has been gradually increasing. The technique for laparoscopic ventral hernia repair depends on the shape, size, location, number, recurrence, and symptoms of the hernia. Computed tomography (CT) is the most accurate method for identifying these factors. Ventral hernia repair begins with an approach to the peritoneal space. Having adequate space to place the mesh is the most important step in surgery. Cosmetic and medical results of primary closure of the hernia margin are superior to those of the bridging technique in laparoscopic ventral hernia repair. However, if primary closure is not possible, the component separation technique can be used to narrow the defect for primary repair of a ventral hernia. Making the abdominal skin flap during the conventional component separation technique can injure the perforator vessels in the abdominal wall, and an injured perforator shuts down the blood supply to the subcutaneous tissue of the abdomen, which then becomes necrotic. To prevent such complications, a perforator-preserving technique can be performed, such as the laparoscopic and posterior component separation techniques. Complications of laparoscopic ventral hernia repair include seroma, hemorrhage, intestinal injury, mesh infection, and recurrence. Mesh infection is one of the most severe complications that sometimes requires reoperation. To prevent infection, it is necessary to minimize contact between the mesh and skin during the surgical procedure.
\end{abstract}

Keywords: Ventral hernia, Incisional hernia, Laparoscopy

This is an Open Access article distributed under the terms of the Creative Commons Attribution Non-Commercial License (http:// creativecommons.org/licenses/by-nc/4.0/) which permits unrestricted non-commercial use, distribution, and reproduction in any medium, provided the original work is properly cited.
Received March 10, 2017

Revised 1st June 19, 2017

2nd August 8, 2017

Accepted August 25, 2017

Corresponding author

Min Chung

Department of Trauma Surgery,

Gacheon University Gil Medical

Center, Namdong-daero 774beongil, Namdong-gu, Incheon 21565,

Korea

Tel: +82-32-460-3010

Fax: +82-32-460-2372

E-mail: yepark26@gmail.com

\section{INTRODUCTION}

The advantages of laparoscopic surgery are decreased the incidence of postoperative mortality and complications such as infections, relatively shorter recovery periods, and lower economic burden. Nevertheless, only a quarter of ventral hernia repairs are performed via the laparoscopic technique in the United States because a proportion of ventral hernias are unsuitable for laparoscopic repair and the complications of laparoscopic repair are more severe than those of open repair. ${ }^{1-3}$ In addition, learning and mastering the laparoscopic hernia repair procedure is technically challenging. However, the use of laparoscopy in ventral hernia repair is steadily increasing. The incidence of complications has decreased with the increased numbers of laparoscopic repairs, and patients' expectations regarding the results of laparoscopic repair also are increasing. The use of the laparoscopic approach to repair ventral hernias is not always successful. If the intestine is injured during laparoscopic repair and resection or suturing of the intestine is required, it may be necessary to switch to the open approach. LeBlanc, who first attempted laparoscopic hernia repair, reported incidences of laparotomy conversion and intestinal injury of $2.4 \%$ and $1.8 \%$, respectively. 


\section{SELECTION OF PATIENTS}

All abdominal wall defects are indications for repair surgery (Fig. 1). Patients with ventral hernia can present with various symptoms. It is important for surgeons to fully understand patient concerns regarding symptoms. Before surgery, surgeons should have sufficient communication with the patient to solve the primary complaint of the patient. Correction of abdominal wall defects should be performed to resolve patient complaints and to prevent potential complications of untreated ventral hernias. The absolute contraindications of laparoscopic ventral hernia repair are severe underlying medical conditions that threaten vital signs during the operation. In patients with liver cirrhosis, preoperative management of ascites is essential. Large amount of ascites cause an interference in the operative field and make it difficult to manage intraabdominal organs during the operation. ${ }^{5}$ It is also necessary to control ascites af-

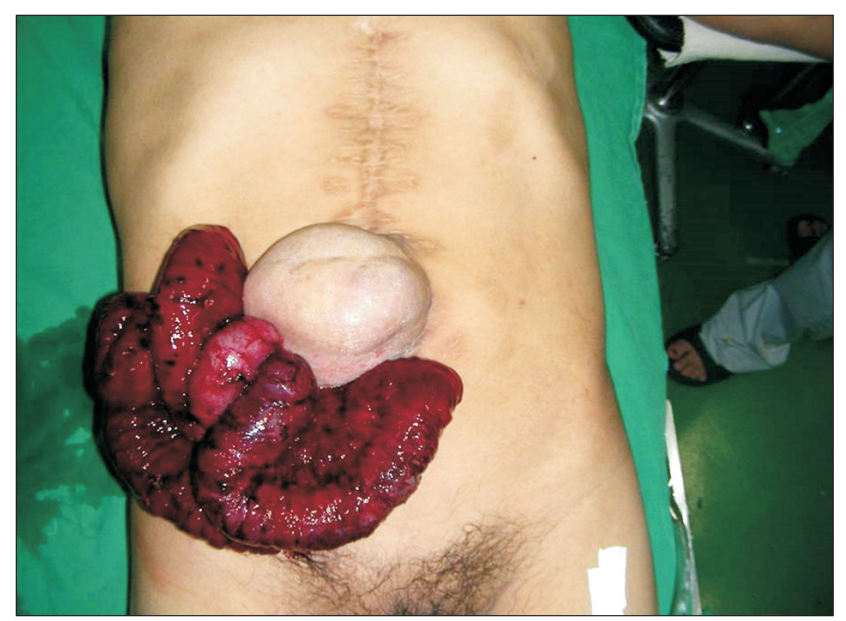

Fig. 1. Ruptured incisional hernia. Image demonstrates the worst sequelae of untreated incisional hernia. ter hernia repair in patients with ascites. Uncontrolled ascites can promote the recurrence of ventral hernia. ${ }^{6}$ If patients with ventral hernia have intraperitoneal infection, this infection should be treated before ventral hernia repair.

\section{DIAGNOSIS OF VENTRAL HERNIA}

Patients with ventral hernia usually present with abdominal pain, discomfort, masses, and symptoms of bowel incarceration and strangulation, such as severe abdominal pain, fever, and obstructive signs. Emergency surgery should be performed if there is incarceration or strangulation, while planned hernia repairs are performed in other cases. Palpation of the abdominal wall defect is a basic procedure to define the patient's condition and plan the operative procedure. The patient lies on the bed with his or her back and soles attached to the bed, knees bent. Then, when the patient lifts his or her head, the abdominal muscles become strained, and the abdominal wall defect is clearly palpable in this position (Fig. 2). The most accurate radiographic diagnostic tool is computed tomography (CT). CT scans reveal the exact size and location of the abdominal wall defect, the degree of atrophy of the abdominal wall muscle around the defect site, and the relationship between the intraperitoneal organs and hernia sac or abdominal wall defect. ${ }^{3}$

\section{THE SURGICAL PROCEDURE OF LAPAROSCOPIC VENTRAL HERNIA REPAIR}

Determining the hernia repair procedure depends on the shape, size, location, number, recurrence, and symptoms of the hernia. ${ }^{8}$

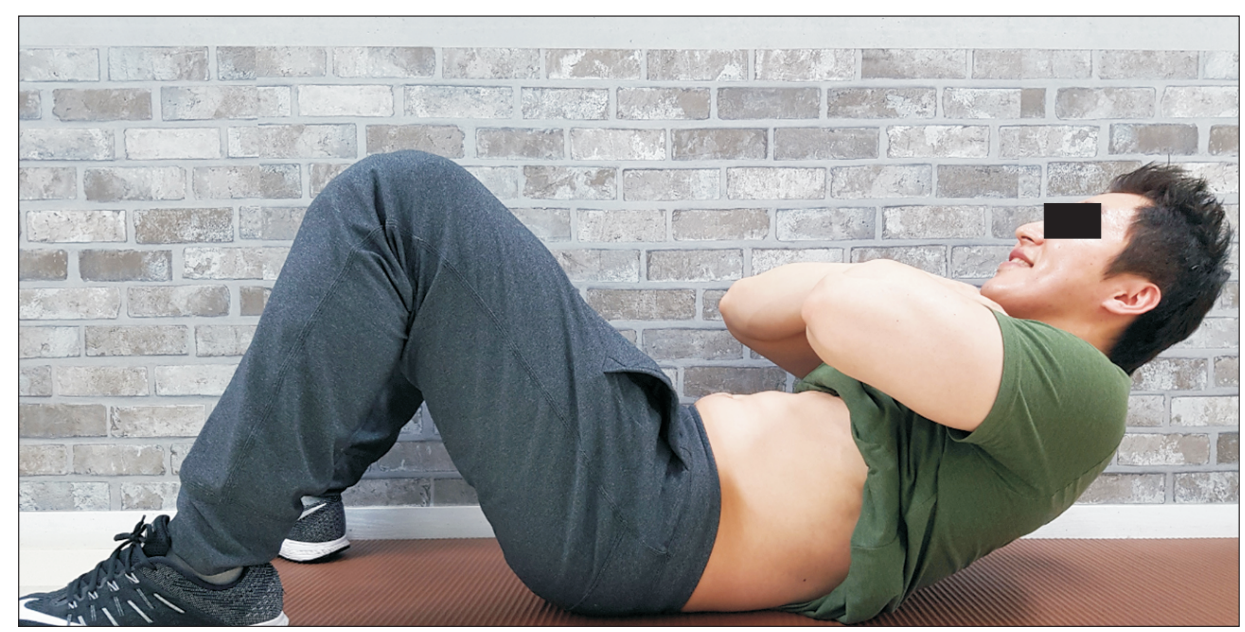

Fig. 2. Straightening of the abdominal wall muscles. The back and soles are positioned on the bed, and the head is elevated. With this position, the abdominal wall muscles are contracted and the defect in the abdominal wall can be easily palpated. 


\section{Approach to the peritoneal cavity}

Normally, the patient is in supine position and we insert three trocars into the left side of the abdomen. It is not necessary to maintain the lithotomy position because it is not approaching the pelvic cavity during ventral hernia repair. We occasionally add one more trocar into the right side of the abdomen. The first trocar is inserted at the tip of the left $11^{\text {th }}$ rib (Fig. 3). This location is least likely to have pathological conditions, such as adhesions. If the spleen is enlarged because of liver cirrhosis or there is a surgical wound in the left upper abdomen, we use the right side to approach the hernia site. In addition, Foley catheter insertion is generally preferred because it takes considerable time to dissect adhesion of hernia sac.

There are various ways to approach the peritoneal cavity. For example, in case of the Hasson technique, a Veress needle with a dilating optic port can be used. Muscles in abdominal wall are separated and a tract is made in the abdominal wall with the finger to enter the peritoneal cavity. We isolate the abdominal muscles and perforate the peritoneum with finger tips to reach the peritoneal cavity. The use of fingers can decrease the possibility of direct injury to the intraabdominal organs. When the peritoneal cavity is approached with direct puncturing with the finger, the finger touches the gutter adjacent to the upper part of the descending colon. Then, a 12$\mathrm{mm}$ balloon trocar is inserted in that site. This trocar has a short intraperitoneal portion and has a better view of the

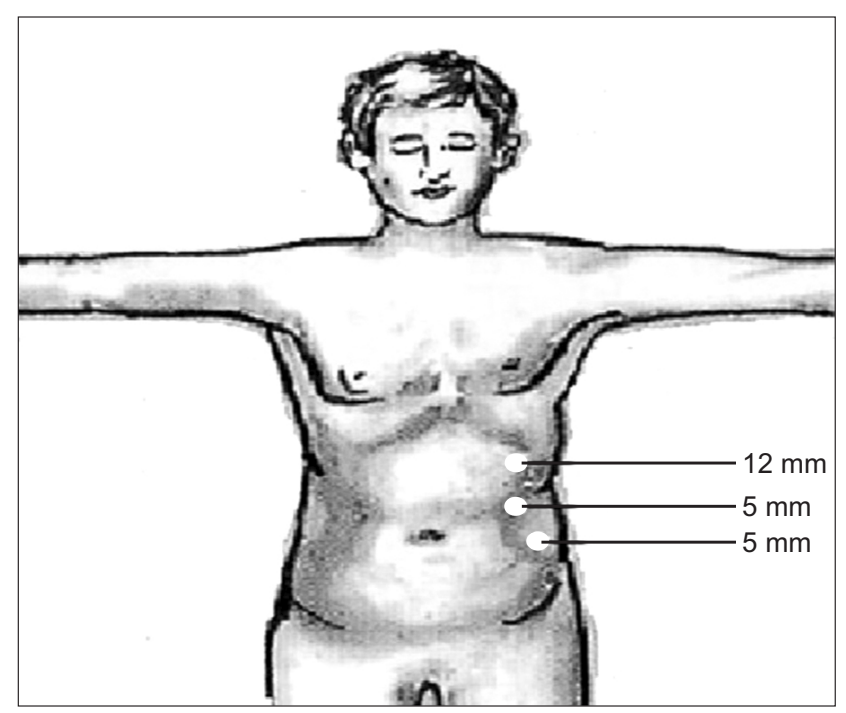

Fig. 3. Trocar plan. Typically, three trocars are used for laparoscopic ventral hernia repair. At the tip of the $11^{\text {th }}$ rib, a 12-mm balloon trocar is inserted. Two 5-mm trocars are inserted at the anterior axillary line and midclavicular line. surgical field. In obese patients with a large amount of intraabdominal fat, fat may accumulate between the descending colon and the abdominal wall. In such situations, the finger may go under the gutter and fail to reach the peritoneal cavity. At this time, it is not possible to determine whether the finger is in the peritoneal cavity or in the retroperitoneal space. In these cases, it is helpful to insert an additional $10-\mathrm{mm}$ trocar on the opposite side of first trocar.

\section{Making space in the peritoneal cavity}

When a trocar is inserted into the peritoneal cavity, the abdominal wall defect and herniated organs should be palpated carefully. If incarcerated or strangulated organs are observed, reduction of these organs performed first (Fig. 4). The easiest way to reduce those herniated organs is to pull the incarcerated or strangulated organs into the abdominal cavity using endoscopic instruments. However, it is not always possible to reduce herniated organs using this pulling method, and the organs tend to be injured easily. Therefore, while pulling the herniated organs into the abdominal cavity, pressure should be applied to the incarcerated or strangulated organs from outside the peritoneal cavity.

The next step is adhesiolysis. There are several methods of dissection of adhesion, including blunt dissection, cold-scissor or hot-scissor dissection, bipolar cautery dissection, and ultrasonic dissection. ${ }^{9}$ Blunt dissection is useful when adhesions are thin and easily separated. However, blunt dissection is likely to cause damage to the small intestine if intestinal adhesions are severe. It is helpful to use endoscopic scissors rather than

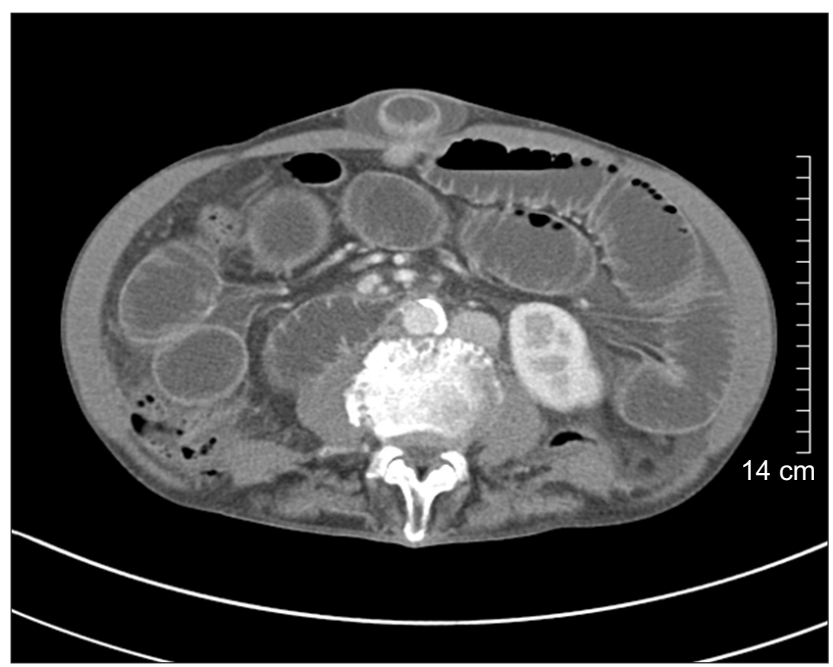

Fig. 4. Incarcerated umbilical hernia. The small bowel is seen herniated through a defect in the umbilicus. The viable mucosa of small bowel indicates absence of strangulation. 
energy devices. Energy devices which use ultrasound generate heat during the procedure, and the heat can damage intraabdominal organs. However, energy devices are very useful for adhesiolysis of the mesentery and blood vessels. During adhesiolysis, surgeons must be very careful to prevent damage to the small bowel. ${ }^{9}$

Adhesiolysis is can performed between the abdominal wall and organs attached to the abdominal wall. In the case of severe adhesion, adhesiolysis is very difficult and intraabdominal organs can be damaged easily. Therefore, during adhesiolysis, part of the abdominal wall should be included to the side of intraabdominal organs..$^{10}$ If organ damage occurs during reduction, the operator should decide whether to continue laparoscopic surgery or to convert to laparotomy. A common complication of adhesiolysis is bleeding..$^{10}$ If bleeding occurs, pressure can be applied directly through the endoscopic device and hemoclips or endoloops can be applied to the bleeding site to control hemorrhage. If the small intestine is clearly separated, hemostasis can be achieved using electrocautery. ${ }^{10}$

\section{Management of intestinal injury during adhesiolysis}

At first, injured organs should be fully assessed. If there is little contamination and the injury is negligible, the operation can be performed as planned. If there is colonic injury or a severe degree of contamination, the bowel injury should initially be managed and hernia repaired few days later. ${ }^{9}$ The treatment of injured organs can be performed by laparoscopic sutures if surgeons can do laparoscopic suturing. If this is not possible, laparotomy conversion should be performed. If necessary, a small abdominal wall incision may need to be placed so that injured organs can be taken out from the abdominal cavity can be sutured. In sesome cases, the use of a biological mesh may be considered to repair hernia site to decrease the chance of infection at hernia site.

\section{Hernia repair}

There are two types of laparoscopic hernia repair. The primary closure technique is suturing the defect in the $a b^{-}$ dominal wall and applying mesh to the suture site. Using a suture passer, the fascial defect is repaired with interrupted suture. The suture materials can be either absorbable or nonabsorbable. We generally use absorbable, monofilament suturing material. Depending on the preference of the surgeon, the barbed thread can be used to repair. The bridging technique is the simple placement of mesh between the defects when primary closure is impossible due to the large size of the defect. According to a comparison of the two methods, the rate of recurrence at 1 year after surgery was 20\% with the bridging

Table 1. Types of meshes with their relative pros and cons

\begin{tabular}{|c|c|c|c|}
\hline Types of mesh & Materials (example) & Pros & Cons \\
\hline \multicolumn{4}{|l|}{ Synthetic } \\
\hline Non-absorbable & $\begin{array}{l}\text { Polypropylene } \\
\text { Polyester } \\
\text { ePTFE/cPTFE }\end{array}$ & $\begin{array}{l}\text { Inexpensive } \\
\text { Low recurrence rate } \\
\text { Relatively less adhesion formation } \\
\text { compared with polypropylene }\end{array}$ & $\begin{array}{l}\text { Infection } \\
\text { Wound pain or discomfort } \\
\text { Visceral adhesion } \\
\text { Higher recurrence rate than polypropylene } \\
\text { Lack of firm tissue incorporation }\end{array}$ \\
\hline Absorbable & $\begin{array}{l}\text { Polyglactin } \\
\text { Polyglycolic acid } \\
\text { Polyglycolic acid/trimethylene carbonate }\end{array}$ & $\begin{array}{l}\text { Lower side effect rate than Non-absorb- } \\
\text { able meshes } \\
\text { Less expensive than biological meshes }\end{array}$ & $\begin{array}{l}\text { Higher recurrence rate than Non-absorb- } \\
\text { able meshes } \\
\text { Insufficient evidence in long term prognosis }\end{array}$ \\
\hline Composite & $\begin{array}{l}\text { Consist of two or more distinct synthetic } \\
\text { component (biface) or non-biface mesh } \\
\text { with temporary coating }\end{array}$ & Less adhesion formation with viscera & Relatively expensive \\
\hline \multicolumn{4}{|l|}{ Biological } \\
\hline Absorbable & $\begin{array}{l}\text { Allogenic human dermis } \\
\text { Xenogenic } \\
\text { Porcine dermis } \\
\text { Porcine intestine } \\
\text { Bovine dermis } \\
\text { Bovine pericardium }\end{array}$ & $\begin{array}{l}\text { Can be applied for infected or intraperi- } \\
\text { toneal field }\end{array}$ & $\begin{array}{l}\text { Expensive, economic burden } \\
\text { High recurrence rate }\end{array}$ \\
\hline
\end{tabular}


method and $0 \%$ with the primary closure method. ${ }^{11}$

\section{Types of mesh}

The mesh for laparoscopic hernia repair must have the characteristics of low fibrosis formation with intraabdominal organs and firm fibrosis formation with the abdominal wall. ${ }^{8}$ Therefore, polypropylene or polyester meshes, which are the typical synthetic meshes that results in tight adhesion with the intraabdominal organs, are not proper for intraabdominal use generally. The commonly used meshes for laparoscopic hernia repair are biological mesh, expanded polytetrafluoroethylene (ePTFE) mesh, and composite mesh which tends to form tight adhesions to the abdominal wall on one side without causing intraabdominal organ adhesion on the opposite side. And the different types of surgical mesh along with their relative pros and cons are listed (Table 1). We generally use non-absorbable, composite meshes in laparoscopic hernia repair but not absorbable meshes. If there is infection or contamination in hernia site, we apply biological meshes.

\section{Location of the mesh}

In laparoscopic abdominal hernia repair, mesh placement methods are generally classified into intraperitoneal onlay mesh (IPOM), transabdominal abdominal preperitoneal (TAPP), and totally extraperitoneal (TEP). IPOM is the most frequent technique used for laparoscopic ventral hernia repair, and the mesh is located just under the peritoneum. Therefore, mesh and organs may contact directly leading to a high rate of adhesions and other surgical complications. The TEP and TAPP procedures are the same as the procedure used for inguinal hernia repair. The mesh is located between the abdominal wall muscle and the peritoneum, without direct contact between the mesh and intraabdominal organs. How ${ }^{-}$ ever, as the operative space is narrow and restricted, there is a high possibility that endoscopic instruments will collide with each other during the operation, thereby increasing the effort and time required to accurately deploy the mesh in the narrow surgical field.

\section{Mesh fixation}

Mesh fixation is an important issue in laparoscopic hernia surgery, because it is challenging procedure and related to hernia recurrence. The mesh should be fixed to the abdominal wall without torsion or bending and with sufficient tightness to cover the abdominal wall defect. ${ }^{12}$ Methods of mesh fixation to the abdominal wall include transfacial sutures, tacks or a combination of both. The tack is a tool to fix the mesh from the abdominal cavity to the abdominal wall and has a fixed depth of approximately $2 \mathrm{~mm}$. Increasing the number of fixation points reduces the maximum force per single tack, but experimental research concluded that the addition of more than three fixation points per $7 \mathrm{~cm}$ of length does not add to fixation strength. The optimal distance between tacks is determined at $1.8 \mathrm{~cm}^{13}$ The position of the mesh can be substantially changed because of mesh fibrosis. To prevent mesh dislocation with one-layer tacking, double crown tacking is used recently. Various kinds of tack are commercialized and absorbable types are also used recently (Table 2). Transfascial fixation is a fixation by suturing the mesh and full layer of the abdominal wall using a suture passer. Suturing over the entire layer of the abdominal wall will cause difficulty in dislocating the mesh after fibrosis formation. However, as pressure is applied to all layers of the abdominal wall, the patient may experience pain in the abdominal wall after surgery. Additionally, fibrin glue is used for mesh fixation but generally be used with tack or transfascial fixation. A significant number of patients suffer from abdominal wall pain after laparoscopic hernia repair due to suture or tack. In an attempt to solve this pain, using fibrin glue is considered as an alternative. However

Table 2. Currently available tacks

\begin{tabular}{|c|c|c|}
\hline & Product name & Material \\
\hline Non- absorbable & $\begin{array}{l}\text { Protack }^{\mathrm{TM}} \text { (Covidien Corp, Mansfield, MA) } \\
\text { PermaFix }^{\mathrm{TM}} \text { (Bard Davol, Maryhill, NY) }\end{array}$ & $\begin{array}{l}\text { Titanium } \\
\text { Polyacetal (polymer based) }\end{array}$ \\
\hline Absorbable* & $\begin{array}{l}\text { SorbaFix }{ }^{\mathrm{TM}} \text { (Bard Davol, Maryhill, NY) } \\
\text { AbsorbaTack }^{\mathrm{TM}} \text { (Covidien Corp, Mansfield, MA) } \\
\text { Permasorb }^{\mathrm{TM}} \text { (Bard Davol, Maryhill, NY) } \\
\text { I-Clip }^{\mathrm{TM}} \text { (Covidien Corp, Mansfield, MA) } \\
\text { Securestrap }^{\mathrm{TM}} \text { (Johnson \& Johnson Medical Limited, Livingston, UK) }\end{array}$ & $\begin{array}{l}\text { Poly(D,L)-lactide } \\
\text { Poly (glycolide-co-L-lactide), } \\
\text { Poly(D,L)-lactide } \\
\text { Poly(D,L)-lactide } \\
\text { Polydiaxonone and } \\
\text { L(-)-lactide/glycolide copolymer }\end{array}$ \\
\hline
\end{tabular}

${ }^{*}$ Average duration time: 12 18 months. 
the long-term prognosis is not certain in mesh fixation. Further research is needed before widespread application in the intraperitoneal mesh fixation alone.

The most important mechanism by which the mesh is fixed to the abdominal wall is fibrosis. However, as fibrosis progresses, the size of the mesh decreases. Therefore, if the portion of the mesh that is wrapped within the defect becomes small, a gap between the abdominal wall defect and the mesh develops and the ventral hernia may recur. Accordingly, the mesh should sufficiently cover the abdominal wall defect. Wrapping the hernia in more than a 5-cm diameter of defect size reportedly decreases the recurrence rate of ventral herniation. ${ }^{14}$

\section{COMPONENT SEPARATION TECHNIQUE}

The operative method for hernia repair is typically chosen according to the size of the abdominal wall defect. If the abdominal wall defect is small, the defect can be completely closed with primary suturing. If the defect is large, it is not possible to seal the defect by primary closure; hence, a mesh is used to cover the abdominal wall defect. Primary closure is closure of the defect site by suturing, while the bridging method is covering the defect with a mesh with or without suturing. Compared with primary closure, the bridging method is associated with a high recurrence rate, short recurrence time, and high postoperative complication rate..$^{15}$ When primary closure is performed, the rectus abdominis muscle is gathered at the abdominal center line and the linea alba is reconstructed. As a result, breathing is improved and physiological pressure of peritoneal cavity is maintained. ${ }^{5}$

\section{Conventional component separation technique}

The component separation technique was first used in clinical practice in 1990 by a Brazilian plastic surgeon, Ramirez. ${ }^{16}$ The main procedure of the technique is the separation of the external oblique muscle from the rectus abdominis muscle along the linea semiluniaris (Fig. 5). When separation is performed, the rectus abdominis muscle on both side moves to the midline, leading to a bilateral gain of $3 \sim 4 \mathrm{~cm}$ on the upper and lower thirds of the abdomen and $8 \mathrm{~cm}$ on the middle third around the umbilicus. As the subcutaneous fat layer and the muscle layer of the abdominal wall should be widely dissected, the blood supply between the abdominal wall muscle layer and subcutaneous fat layer is likely to be interrupted. Therefore, complications, including the interruption of blood supply to a skin flaps, infection, hematoma, and seromas may occur (Fig. 6).

\section{Perforator-preserving component separation technique}

Through a separate incision, dissection is made only for the linea semilunaris area, and the rectus abdominis muscle and the external oblique muscle are separated. This procedure preserves the perforator vessel that connects the deep and superficial inferior epigastric artery around the navel, and dis-
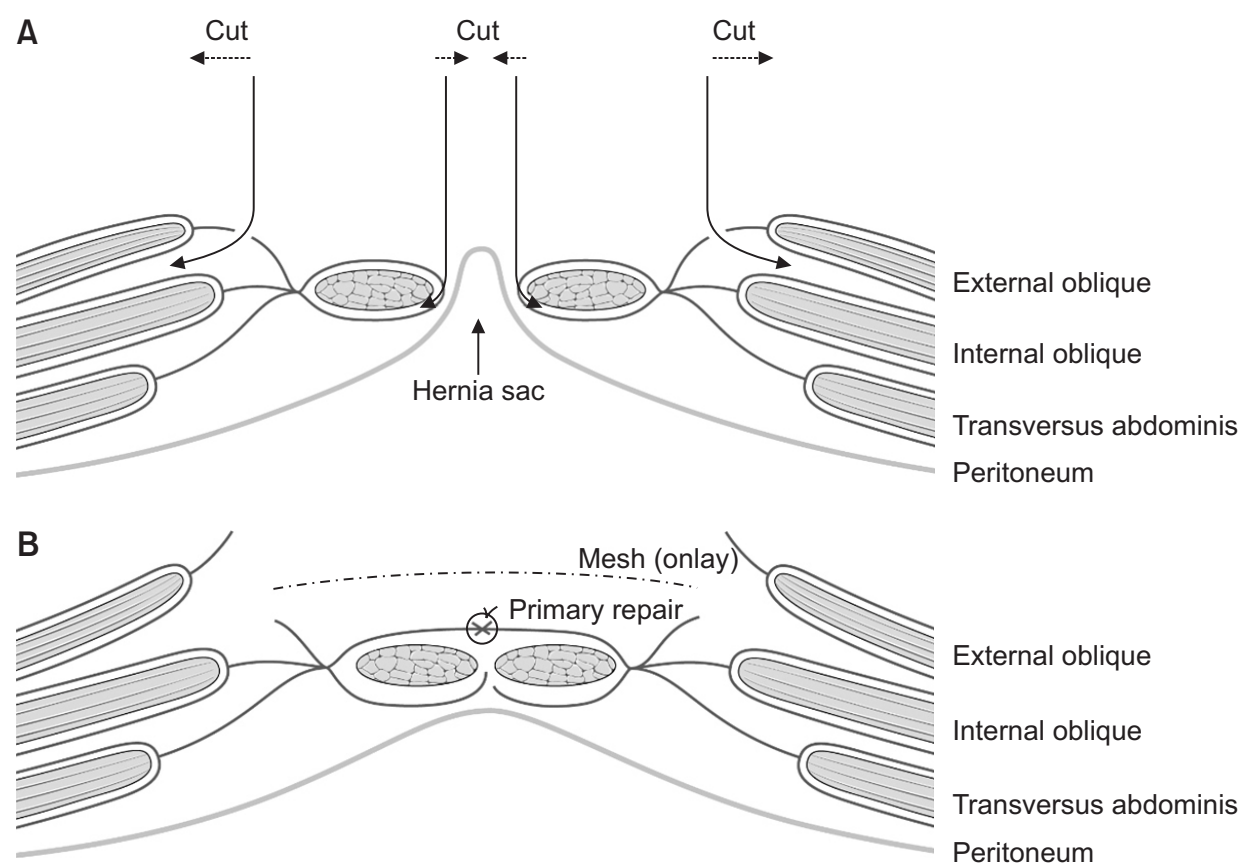

Fig. 5. Component separation technique. The first procedure of the technique is the separation of the external oblique muscle from the rectus abdominis muscle along the linea semiluniaris (A). When separation is performed, the rectus abdominis muscle on both side moves to the midline closer, the primary repair can be done with less tension (B). 


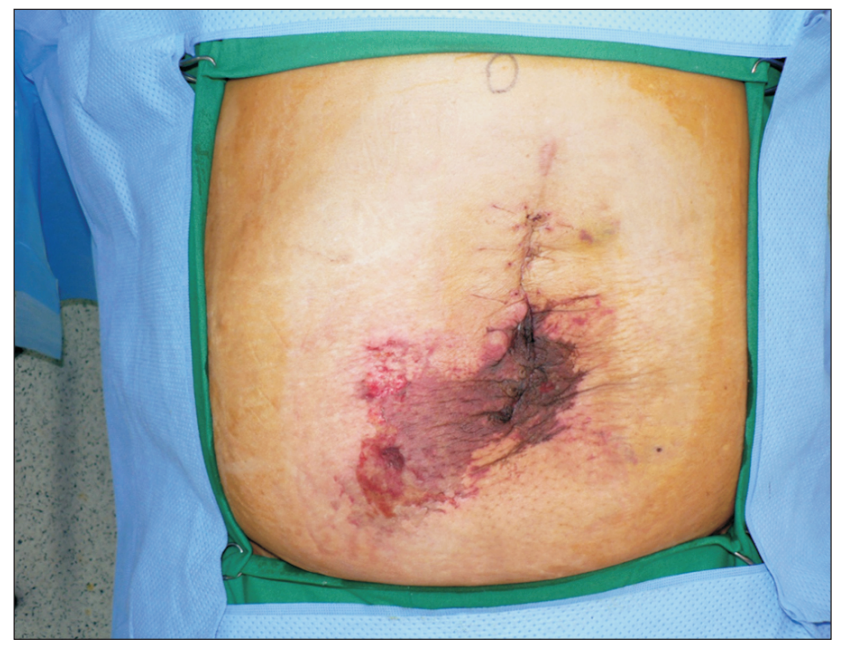

Fig. 6. Skin necrosis after the use of the conventional anterior component separation technique. The blood supply via the perforator around the umbilicus has been interrupted, leading to the development of skin necrosis.

section of the subcutaneous layer can thus be decreased. ${ }^{17}$ This is designed to prevent interruption of the blood supply from epigastric artery to the abdominal skin flap resulting from the conventional separation technique.

\section{Posterior component separation technique}

Two procedures described above use the back space of the rectus abdominis muscle. However, there are many restrictions to use this space because it is narrow, and it is not possible to insert a large mesh into this space. When the posterior fascia of the rectus abdominis muscle is separated from the rectus muscle, the transverse abdominis muscles attached to the posterior fascia can be identified in the upper third level of the rectus abdominis muscle. Here, when the transverse abdominis muscles are separated, the posterior fascia of the rectus muscle and peritoneum is separated into one layer providing access to the preperitoneal space. A large space is created that connects to the diaphragm superiorly, the pelvic cavity inferiorly, and the retroperitoneum on the bilateral side. Thus, a large mesh can be used to reinforce a wide range of defects. This is a completely different procedure from the two surgical procedures described previously. ${ }^{18}$

\section{Laparoscopic component separation technique}

The laparoscopic component separation technique is a perforator-preserving technique using a laparoscopic device. ${ }^{1920}$ Once the laparoscopic device is used to create a space between the external and internal oblique muscles, the location of the camera can be determined according to the use of laparoscopic component separation technique only or the performance of complete laparoscopic ventral hernia repair. The semilunar line is located where the external oblique muscle and the rectus abdominis muscle meet. The linea semilunaris is marked on the skin, which helps to identify the lateral border of the rectus abdominis muscle from the outside. The maximal effect of perforator preservation in the laparoscopic component separation technique appears 2 3 days after component separation procedure. Then, the ventral hernia can be repaired either by laparotomy or the laparoscopic approach.

\section{COMPLICATIONS}

The complications of laparoscopic abdominal hernia repair can be divided into a major category, including hemorrhage, intestinal injury, mesh infection, and recurrence, and a minor category, including seroma, ileus, and wound pain. ${ }^{9}$ Seromas occur after hernia repair in almost all patients. The use of an abdominal binder at the surgical site decreases the incidence of seromas. The most common bacteria found in mesh infections are Staphylococcus aureus, Staphylococcus epidermidis, and Escherichia coli. ${ }^{21}$ Except for E. coli, the other bacterial causes of infection are skin microbes. Therefore, it is necessary to minimize contact between the mesh and the skin during the surgical procedure, and to minimize the transfer of bacteria to the mesh. Ioban drapes (3M, Maplewood, Minnesota, USA) are used during hernia repair to decrease contact between the skin and mesh.

\section{CONCLUSION}

The important factors to be considered in laparoscopic ventral hernia repair are securing the space to apply the mesh, preventing injury to intraabdominal organs, and preventing recurrence and postoperative complications. If the ventral hernia defect is large, a proper space to deploy mesh can be secured by using the conventional or posterior component separation technique. If the blood supply between the abdominal wall muscle layer and subcutaneous fat layer is likely to be interrupted during the component separation procedure, the perforator-preserving component separation technique can be performed. However, in the perforator-preserving component separation technique, it is not always possible to have adequate space to deploy a mesh. In that case, the posterior or laparoscopic component separation technique may be used to create a large space to deploy mesh and expect to have good prognosis. 


\section{REFERENCES}

1) Hwang CS, Wichterman KA, Alfrey EJ. Laparoscopic ventral hernia repair is safer than open repair: analysis of the NSQIP data. J Surg Res 2009;156:213-216.

2) Savitch SL, Shah PC. Closing the gap between the laparoscopic and open approaches to abdominal wall hernia repair: a trend and outcomes analysis of the ACS-NSQIP database. Surg Endosc 2016;30:3267-3278.

3) Slater NJ, Knaapen L, van Goor H. Abdominal wall defects: pathogenesis, prevention and repair. Surgery (Oxford) 2015;33:206-213.

4) LeBlanc KA. Incisional hernia repair: laparoscopic techniques. World J Surg 2005;29:1073-1079.

5) Singh NK, Khalifeh MR, Bank J. Abdominal wall reconstruction. In: Neligan PC, Song D, editors. Plastic surgery: Lower extremity, trunk and burns; vol. 4. 3rd ed. London; New York: Elsevier Saunders; 2013. p.279-296.

6) Yu BC, Chung M, Lee G. The repair of umbilical hernia in cirrhotic patients: 18 consecutive case series in a single institute. Ann Surg Treat Res 2015;89:87-91.

7) Melvin WS, Renton D. Laparoscopic ventral hernia repair. World J Surg 2011;35:1496-1499.

8) Tobler WD, Jr., Itani KM. Current Status and Challenges of Laparoscopy in Ventral Hernia Repair. J Laparoendosc Adv Surg Tech A 2016;26:281-289.

9) LebBlanc K. Laparoscopic Incisional and Ventral Hernia Repair. In: Jones DB, editor. Hernia. Philadelphia: Wolters Kluwer Health/Lippincott Williams \& Wilkins; 2013. p.353-365.

10) Philipp BJ. Laparoscopic ventral and incisional hernia repair. In: Cameron JL, Cameron AM, editors. Current surgical therapy. Philadelphia, PA: Elsevier Saunders; 2011. p.1214-1221.

11) Mitura K, Skolimowska-Rzewuska M, Garnysz K. Outcomes of bridging versus mesh augmentation in laparoscopic repair of small and medium midline ventral hernias. Surg Endosc 2017;31:382388.

12) Alexander AM, Scott DJ. Laparoscopic ventral hernia repair. Surg Clin North Am 2013;93:1091-1110.

13) van't Riet M, de Vos van Steenwijk PJ, Kleinrensink GJ, Steyerberg EW, Bonjer HJ. Tensile strength of mesh fixation methods in laparoscopic incisional hernia repair. Surg Endosc 2002;16:17131716.

14) LeBlanc K. Proper mesh overlap is a key determinant in hernia recurrence following laparoscopic ventral and incisional hernia repair. Hernia 2016;20:85-99.

15) Booth JH, Garvey PB, Baumann DP, et al. Primary fascial closure with mesh reinforcement is superior to bridged mesh repair for abdominal wall reconstruction. J Am Coll Surg 2013;217:9991009.

16) Heller L, McNichols CH, Ramirez OM. Component separations. Semin Plast Surg 2012;26:25-28.

17) DeNoto $G$, Israeli R. Periumbilical perforator sparing components separation. In: Rosen MJ, editor. Atlas of abdominal wall reconstruction. Philadelphia: Elsevier; 2012. p.139-170.

18) Novitsky YW, Elliott HL, Orenstein SB, Rosen MJ. Transversus abdominis muscle release: a novel approach to posterior component separation during complex abdominal wall reconstruction. Am J Surg 2012;204:709-716.

19) Rosen MJ, Jin J, McGee MF, Williams C, Marks J, Ponsky JL. Laparoscopic component separation in the single-stage treatment of infected abdominal wall prosthetic removal. Hernia 2007; $11: 435-440$.

20) Lowe JB, Garza JR, Bowman JL, Rohrich RJ, Strodel WE. Endoscopically assisted "components separation" for closure of abdominal wall defects. Plast Reconstr Surg 2000;105:720-729; quiz 730.

21) Engelsman AF, van der Mei HC, Ploeg RJ, Busscher HJ. The phenomenon of infection with abdominal wall reconstruction. Biomaterials 2007;28:2314-2327. 